\title{
Development and Performance of a Filter Radiometer Monitor System for Integrating Sphere Sources
}

\author{
Leibo Ding ${ }^{\mathrm{a}}$, Matthew G. Kowalewski ${ }^{\mathrm{b}}$, John W. Cooper ${ }^{\mathrm{a}}$, Gilbert R. Smith ${ }^{\mathrm{a}}$, \\ Robert A. Barnes ${ }^{c}$, Eugene Waluschka ${ }^{\mathrm{d}}$, and James J. Butler ${ }^{\mathrm{d}}$ \\ ${ }^{\mathrm{a}}$ SigmaSpace Corporation, Lanham, MD 20706

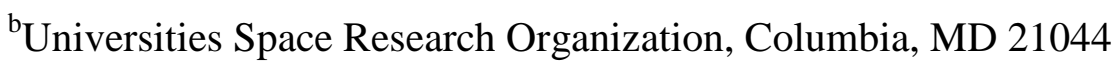 \\ 'Science Applications International Corporation, Beltsville, Maryland 20705 \\ ${ }^{\mathrm{d}}$ NASA Goddard Space Flight Center, Greenbelt, MD 20771
}

\begin{abstract}
The NASA Goddard Space Flight Center (GSFC) Radiometric Calibration Laboratory (RCL) maintains several large integrating sphere sources covering the visible to the shortwave infrared wavelength range. Two critical, functional requirements of an integrating sphere source are short and long-term operational stability and repeatability. Monitoring the source is essential in determining the origin of systemic errors, thus increasing confidence in source performance and quantifying repeatability. If monitor data falls outside the established parameters, this could be an indication that the source requires maintenance or re-calibration against the National Institute of Science and Technology (NIST) irradiance standard. The GSFC RCL has developed a Filter Radiometer Monitoring System (FRMS) to continuously monitor the performance of its integrating sphere calibration sources in the 400 to $2400 \mathrm{~nm}$ region. Sphere output change mechanisms include lamp aging, coating (e.g. $\mathrm{BaSO}_{4}$ ) deterioration, and ambient water vapor level. The FRMS wavelength bands are selected to quantify changes caused by these mechanisms. The FRMS design and operation are presented, as well as data from monitoring four of the RCL's integrating sphere sources.
\end{abstract}

Subject terms: integrating sphere, calibration, stability, radiometer 


\section{Introduction}

Two critical requirements of any optical calibration source are short and long-term operational stability and repeatability. Without continuous monitoring of the source, short-term source instabilities may not be noticed and could possibly be incorrectly attributed to instabilities or transients in the instrument being calibrated. Also, long-term source instabilities may never be detected until the source output diverges grossly, making repeatability difficult to establish.

The degradation of sphere radiant output is wavelength dependent. Commercial sphere source output monitoring devices usually consist of a single silicon detector sometimes equipped with a broadband photopic filter mounted at a port on the sphere. As a result of the broadband wavelength response of the silicon detector, only gross sphere output degradation or variability is detected. These monitoring devices are insufficient to detect the wavelength selective degradation that would affect the individual narrow band wavelengths of radiometers being calibrated by the source. Examples of the importance of monitoring short and long term stability and repeatability can be found in the historical applications of integrating spheres in the calibration of satellite Earth remote sensing instruments ${ }^{1-5}$. For many of these applications, the calibration of the integrating sphere is performed well before being used in the calibration of the satellite instrument. It is erroneous to assume that the integrating sphere output is unchanged between the times of its calibration and its use. For these cases, in order to realize radiance calibration uncertainties of 3 to $5 \%(k=1)$ typical of NASA's Earth Observing System (EOS) satellite instruments operating from 400nm to 2500nm, the output of integrating sphere sources must be continuously monitored using stable radiometers operating at or across the satellite instrument wavelengths.

The GSFC RCL maintains and operates a suite of integrating sphere sources capable of meeting the radiance and irradiance calibration requirements of satellite instruments viewing dark ocean to bright clouds. The integrity of the GSFC RCL integrating sphere sources is maintained with NIST traceable calibrations. Sphere source output radiance is slightly variable and unstable as a function of the type and condition of the internal reflectance surface, the aging characteristics of the internal lamps, and sphere ambient relative humidity and temperature. As a result, there exists a need to continuously monitor the stability of the sphere source output over their wavelength operating range of 400 to $2400 \mathrm{~nm}$. The production of high quality, accurate sphere source data requires that this variability be monitored and, if necessary, used to apply a correction factor to the data. The GSFC 
RCL FRMS ${ }^{6}$, whose development and performance are now fully described in this paper, is an optically simple yet versatile eleven band filter radiometer used in the RCL to monitor sphere stability and repeatability. In combination with additional instrumentation, the FRMS is also shown to be able to measure sphere uniformity and to characterize the polarization properties of sources and optics.

\section{FRMS Description}

A dedicated, optically simplistic, electronically stable detector and optical filter monitoring system best serves the need to document the degradation and variability of the sphere output. The GSFC integrating sphere source FRMS is such a system and provides time line data of the sphere output stability and variability. A greater confidence in the integrity of the sphere output will thereby be maintained through use of the FRMS. The FRMS is a filter wheel radiometer and is a computer controlled, software operated unit. A block diagram of the FRMS is shown in Fig. 1. The optical input of the FRMS is a Gershen tube with apertures to define the field of view (FOV). The FOV is defined by the incident light (i.e. dashed lines) shown in Fig. 1. Two critical requirements of the FRMS are short and long-term operational stability and repeatability. The FRMS sphere source data is an asset in determining the origin of systematic errors, thus increasing confidence in sphere source performance and in quantifying source repeatability. If monitor data falls outside established parameters, this is an indication that the source may require maintenance or re-calibration against the standard. Several FRMS optical filter wavelength bands have been selected to be in those areas of scientific interest that are sensitive to the mechanisms of common degradation of the sphere output.

The heat from the lamps of sphere sources naturally causes the sphere to be elevated in temperature to the extent that this is detrimental to the performance and stability of the FRMS detector, optical assembly, and electronics. Therefore, directly mounting the FRMS to a small aperture on the sphere source is impractical at this time. The FRMS must be thermally isolated from the sphere. Thermal isolation from the sphere can be realized by placing a fiber optic bundle or a light pipe between the sphere and the FRMS entrance aperture. These methods are currently under investigation. In lieu of this, data has been gathered with the FRMS placed on a table, viewing the main aperture of the sphere at a distance of $50 \mathrm{~cm}$. The FRMS has been tested and characterized and 
the results are presented. Fig. 2 shows the FRMS monitoring an integrating sphere in this configuration.

\section{Detector Heads}

An ultraviolet (UV) enhanced silicon (Si) detector is used for the FRMS in the wavelength range of 400 to $1100 \mathrm{~nm}$. A second FRMS employs an extended-InGaAs detector and is used for the wavelength range of 1100 to 2400nm. Also, two additional detectors are available for use with the FRMS: a two-element "sandwich” detector composed of a Si element and an InGaAs element to cover the spectral range of 320 to $2570 \mathrm{~nm}$ and a Ge detector for the spectral range of 800 to $1800 \mathrm{~nm}$.

The two-element detector is a hybrid sandwich-style detector containing an infrared-transmitting Si photodiode mounted over an InGaAs PIN photodiode configured along the same optical axis. The benefits of using the two-element detector are the wide spectral response range and the single optical axis configuration for both detectors. The drawbacks are lower sensitivities and a smaller active area for the InGaAs detector, making it an undesirable choice for the applications with a chopper. Table 1 is a data sheet for these detectors. A transimpedance amplifier (i.e. current-to-voltage converter) is used for the pre-amp. The selection of the operational amplifier (OA) is based on low noise, low drift, low bias (i.e. voltage and current), low input capacitance, high gain, and high resistance. To reduce the noise, "the simpler is better" principle is applied for the pre-amp: the detector is set as close as possible to the input of the pre-amp; and there is no selection switch (e.g. sensitivity selection, bias cancellation, detector type selection). The pre-amp contains an OA, a precision resistor, and a tiny-value capacitor for frequency compensation. Each detector has a dedicated preamp with different resistor and capacitor. The voltage signal from the OA is directly sent to a Digital Multi-Meter (DMM) HP 3458A without any modification. A second-stage amplifier can be used, but it is not necessary in this application. The detector and the pre-amp are mounted in a metal cylinder-shaped container as shown in Fig. 3.

\section{Filters and Filter Wheel}

One opaque blocking element and 11 band-pass filters of $2.54 \mathrm{~cm}$ diameter were installed for the FRMS initial testing and characterization. The filter center wavelengths/bandwidths in nm are as 
follows: 410/10, 440/10, 460/10, 640/10, 840/10, 1050/15, 1240/20, 1380/20, 1640/20, 2130/30, and 2210/25. All filters are stock items. Filter selection is critical and the initial filter bands were selected to measure and bracket spectral scientific regions of interest. The $840 \mathrm{~nm}$ and $1050 \mathrm{~nm}$ bands are used to monitor the source output spectral peaks; the $1240 \mathrm{~nm}, 1380 \mathrm{~nm}$ and, $1640 \mathrm{~nm}$ bands bracket the first significant water absorption band; while 2130nm and 2210nm bands are used to monitor the infra-red spectral characteristics of the sources. Past experience in operating quartz tungsten halogen lamps of the type used as RCL sources suggests that lamp degradation is most noticeable in the UV region and this drove selection of the 410nm, 440nm and 460nm bands to monitor lamp degradation. Also the 410nm and 440nm bands are widely used by other standard transfer radiometers, such as the SeaWiFS Transfer Radiometer (SXR) and the Visible Transfer Radiometer (VXR) and as MODIS bands. Note that the initial filters are exchangeable with other band-pass filters according to applications. Fig. 4 shows the twelve position rotating filter wheel.

\section{FRMS Electronic Noise Level and Stability}

The FRMS electronic noise includes the dark noise from the detector photodiode and the operational amplifier noise and offset ${ }^{7}$. To measure this noise, the opaque blocking element is used to cover the detector's input window; and the output from the operational amplifier (i.e. the dark signal) was recorded. The stability of the dark signal is a direct indication of the stability of the detector head, that is, the combination of the detector and pre-amp.

The dark signal was measured along with other filters' signals and was used as the reference signal subtracted from the optical filter signals. Fig. 5 shows a typical dark signal plot. The twoelement detector, the K3413-09 thermo-electrically cooled $\mathrm{Si}+$ InGaAs detector was used to acquire these data using the RCL large integrating sphere called "Hardy". Details on this and other integrating spheres used in this study are provided in the next section of this paper. In this measurement of dark signal, the sphere was turned on with all 16 lamps, then dropped to the 12 lamp level, and finally was turned off. During this period, the ambient temperature increased from $75^{\circ} \mathrm{F}$ to $98^{\circ} \mathrm{F}$; but the dark signals remained stable.

\section{Sphere Response Stability Measurements}


Four integrating sphere sources were used to evaluate and characterize the FRMS. They are listed below:

- GSFC 1.83m diameter integrating sphere, dubbed “Hardy,” with a barium sulfate $\left(\mathrm{BaSO}_{4}\right)$ interior coating and a 25.4cm diameter exit aperture. This sphere is equipped with 16 baffled 200W quartz tungsten halogen lamps.

- GSFC 91.4cm diameter polytetrafluoroethylene (PTFE) integrating sphere, dubbed "Slick," lined with Spectralon ${ }^{\mathrm{TM}}$ and a 25.4cm diameter exit aperture. This sphere is equipped with 16 unbaffled 45W quartz tungsten halogen lamps.

- GSFC 50.8cm diameter PTFE integrating sphere, dubbed “Venti," lined with Zenith" ${ }^{\mathrm{TM}}$ coating and a $20.3 \mathrm{~cm}$ diameter exit aperture. This sphere is equipped with 4 quartz tungsten halogen lamps: two $150 \mathrm{~W}$, one $300 \mathrm{~W}$ and one $75 \mathrm{~W}$ with an attenuator.

- GSFC 101.6cm diameter PTFE integrating sphere, dubbed “Grande," lined with Zenith" ${ }^{\mathrm{TM}}$ coating and a $25.4 \mathrm{~cm}$ diameter exit aperture. This sphere is equipped with 8 baffled $300 \mathrm{~W}$ quartz tungsten halogen lamps and one 150W quartz tungsten halogen lamp with a variable attenuator.

- NASA Ames Research Center (ARC) $15.2 \mathrm{~cm}$ diameter radiance standard OL-455 with a $\mathrm{BaSO}_{4}$ interior coating and a $3.8 \mathrm{~cm}$ diameter exit aperture dubbed "ARS455". This sphere is equipped with a single $150 \mathrm{~W}$ quartz tungsten halogen lamp.

Other instruments used to characterize the FRMS include:

- The Shuttle Solar Backscatter Ultraviolet (SSBUV) instrument: The SSBUV is a refurbished Solar Backscatter Ultraviolet/2 (SBUV/2) instrument of the type flown on the NOAA Polar Orbiting Environmental Satellites (POES). This instrument was flown eight times on the Space Shuttle between 1989 and 1996. The instrument is a scanning double Ebert-Fastie holographic spectrometer with $1.1 \mathrm{~nm}$ band-pass operating from 240 to $405 \mathrm{~nm}$.

- An Avantes Spectrometer, AvaSpec-2048-USB2 with UA-300 lines/mm grating, 50 $\mu$ m slit, 2048 pixel CCD detector, 16 bit ADC, a spectral range of 200 to 1100nm, and a spectral resolution of $2.4 \mathrm{~nm}$.

- Two Analytical Spectral Devices (ASD) FieldSpec3 Full Range spectrometers with spectral ranges of 350-2500nm.

\subsection{Short-term stability measurements}


Three of the GSFC integrating spheres were monitored by the FRMS and the results are plotted in Fig. 6 to 8.

In Fig. 6, the two upper plots, a and b, were from measurements taken on 10/16/2008. At the same time, an ASD was used to make a comparison with the FRMS and the results are shown in Fig. 6c. The results show reasonable agreement with each other. Figure $6 \mathrm{~d}$ shows the results from the FRMS equipped with an extended InGaAs detector on another day.

In Fig. 7 (i.e. Slick), the two upper plots, a and b, show two measurements from the FRMS Si and Ge detectors. At the same time, the Avantes Spectrometer was used to cross calibrate the FRMS and its results are plotted as Figure 7c. The cross calibration also shows consistency between the FRMS and the Avantes Spectrometer. Figure 7d shows the results from the FRMS equipped with an extended InGaAs detector on another day.

In Fig. 8 (i.e. Venti), the plots show the stability measurement results from the FRMS equipped with $\mathrm{Si}$ and Ge detectors.

Table 2 shows the calculated noise equivalent radiance (NER) using the Slick integrating sphere with all lamps illuminated. The measurement uncertainty and the related NER associated with the FRMS is determined by observing the signal level and the maximum amplitude of the dark signal for each channel. The signal level is related to radiance level. The maximum amplitude of the dark signal can be obtained by observing the dark signal plots as shown in Figure 5. The maximum amplitude of the dark signals associated with the $\mathrm{Si}, \mathrm{Ge}$ and extended InGaAs detectors are $4 \mathrm{uV}, 40 \mathrm{uV}$ and $6 \mathrm{uV}$ respectively. The FRMS uncertainty can be calculated as: maximum amplitude/signal level. The NER can be obtained by multiplying the uncertainty by the radiance level.

\subsection{Measurement comparison with SSBUV}

On February 7, 2008, a measurement comparison between the FRMS and the SSBUV was performed at the GSFC Code 613.3 Radiometric Calibration and Development Laboratory. The ARS455 sphere from NASA AMES was used as the radiance standard. Since there was no overlapping spectral range between these two instruments, two filters, 360/10 and 380/10, were selected to replace two of the initial optical band-pass filters in the FRMS. The data at 360nm and 380nm were compared between the FRMS and the SSBUV; and results were plotted in Fig. 9. 


\subsection{Long-term stability measurements}

One of the capabilities of the FRMS is to monitor a sphere's long-term stability. Since the FRMS design and operational mode have not been completely finalized, a long-term database has not been acquired to validate this function. Once the FRMS is finalized, data will be acquired every month or after every 100 hours of usage and the results will be plotted to show the trends. These data will provide insight into the degradation and variation of sphere lamps and interior coatings. Sphere data acquired over several days with identical warm-up times are shown in Fig. 10. The data taken on October 17, 2009 are used as the reference; data taken after that date are compared to that reference. The determination of long-term stability requires long data accumulation times. Therefore, these plots are provided here for demonstration only.

Further examination of the day-to-day data in Fig. 10 revealed a significant change in radiance of $0.5 \%$ to $1 \%$. We have attributed the cause of this additional uncertainty to be the relative instability of the lamp power supplies. Vendor data indicates power supply current accuracy to better than $12 \mathrm{~mA}$ (i.e. $0.17 \%$ in current). Our laboratory measurements indicate that a change of $1.5 \mathrm{~mA}$ in power supply current (i.e. $0.025 \%$ ) will cause a change of $0.1 \%$ in sphere output radiance at a wavelength of $1000 \mathrm{~nm}$.

\section{Other Applications}

Since the FRMS has very low noise level and credible stability, in addition to its initial usage, it also can be used in other applications.

\subsection{Source Aperture Mapper (SAM)}

The radiance uniformity of the source output is a critical characteristic for calibration, especially when the instrument is sensitive to radiance with a small field of view (FOV). The FRMS equipped with a small FOV aperture can be used to map the uniformity of the source output. An X-Y translation stage was refurbished and used to raster scan across a two-dimensional plane parallel to the aperture plane. The FRMS is mounted on the platform of the translation stage shown in Fig. 11. The scanning resolution is determined by the FOV of the FRMS and distance from the source output aperture to the detector in the FRMS. Since the distance is predetermined (i.e. $50 \mathrm{~cm}$ ), the FOV of 
the FRMS solely controls the scanning resolution. A FOV of $1.91^{\circ}$ is used to achieve the $1 \mathrm{x} 1 \mathrm{~cm}$ scanning resolution.

In order to make more accurate measurements, a reference scanning method was used shown in Fig. 12. Using the center of the output aperture as the reference point $\mathrm{R}$, the scanning sequence is Column $1 \rightarrow \mathrm{R} \rightarrow$ Column $2 \rightarrow \mathrm{R} \rightarrow$ Column $3 \rightarrow \mathrm{R}$ and so forth. The measurement values at the reference point $\mathrm{R}$ were linearly interpolated and assigned to other scanning points as references. In this way, the relative uniformity at each scanning point was calculated against the reference point, R.

Fig. 13 shows the uniformity plots of the four GSFC integrating spheres. Note that the "Venti" sphere has a non-symmetric lamp positioning around its exit aperture, and its output aperture size is relatively large compare to its body size. The Venti sphere uniformity shows a stratified pattern from the 9 o'clock position, where the $300 \mathrm{~W}$ lamp is located, to the 3 o'clock position, where the $75 \mathrm{~W}$ lamp is located.

\subsection{Polarization Sensitivity Measurement}

In support of the characterization of the NPOESS Preparatory Project (NPP) program's Visible Infrared Imager Radiometer Suite (VIIRS), preliminary polarization measurements on a flat sheet polarizer were performed using the FRMS. The measurement system set up is shown in Figure 14. The components shown in Fig. 14 are as follows from left to right: the $30.5 \mathrm{~cm}$ diameter sphere source, the polarizer rotating stage, a fused silica optical flat and the FRMS. The integrating sphere is used as a stable light source. The polarizer sheet under test is mounted on the rotating stage.

An initial baseline measurement was performed without the polarizer sheet and the optical flat. Then the polarizer was attached to the rotating stage, and the polarization component from the sphere source was measured while the polarizer was rotated. The results of this measurement indicated that the sphere source light was linearly polarized at the $0.01 \%$ to $0.001 \%$ level with the largest percentage of polarization at the shortest, 360nm, wavelength. Finally, the optical flat was inserted and tilted $30^{\circ}$ to the incident light. The resulting light intensity variations are shown in Fig. 15. The difference between these measurement data and the theoretical Fresnel prediction was less than $0.2 \%$ from 410 to $870 \mathrm{~nm}$ as shown in Table 3. For this calculation, the effect of multiple reflections within the optical flat was removed.

\section{Modifications for System Stability}


The FRMS performs satisfactorily with Si, Ge, and standard InGaAs detectors. However, the TE cooled extended-InGaAs detector has a dark signal instability related to the environmental temperature change. Fig.16 shows the instability of the dark signal from the two-stage TE cooled extended-InGaAs detector. A reduction in the instability of the dark signal has been effected by enlarging the FOV to $6.4^{\circ}$, thereby introducing more signal. To eliminate the environmentally induced slow changes, including the temperature instability, the FRMS was modified from DC mode to AC mode using a chopper and lock-in amplifier. A chopper was added in front of the filters in the filter wheel assembly shown in Fig. 17. In this configuration, the output signal is directly sent to a lock-in amplifier for data acquisition and recording. After the modification was complete, several measurements were made with an extended-InGaAs detector to compare the FRMS results in DC mode and AC mode. These are shown in Fig. 18.

Figure 18 contains 4 plots from two different lamp levels; the two plots in the upper row are from full-lamp level measurements, and the two plots in the lower row are from a lower lamp level. In these measurements, the same extended InGaAs detectors and FOV were used to provide a viable comparison. The plots show consistency in the stability trend, but they also show some differences. All the DC mode signals tend to ramp up at the beginning and drop off at the end, as compared to the AC mode. This can be explained as follows. When the sphere Grande was illuminated producing more than 2,000 Watts of additive lamp power, the room temperature gradually increased and eventually caused the dark signal of the extended InGaAs detector to increase, as shown in Fig. 16. Since the measurement results under DC mode require subtraction of the dark signal, it produced DC signals that show a higher beginning and lower end. In addition, a vertical comparison of the plots with same wavelengths under different lamp levels indicates that, at stronger light level, the AC mode does not provide much advantage in the signal-to-noise performance. However, after reducing the light level, the DC mode signal gets noisier than the AC mode on the same scale. We have concluded that, under low light levels, a larger active-area detector working in AC mode will provide better signal-to-noise performance than the DC mode.

\section{Conclusions}

In the absence of a source monitor, the short and long-term variability of integrating sphere output cannot be quantified. Without this knowledge, the sphere cannot be used as a viable 
calibration transfer device, since its stability and repeatability is questionable. The use of a viable multi-spectral source monitor is therefore required for all integrating sphere sources used as calibration standards. The FRMS instrument, as a multi-band filter radiometer operating in DC mode, is capable of monitoring changes in the output of integrating sphere sources at the 0.008 to $0.013 \%$ level over the 410 to $2130 \mathrm{~nm}$ wavelength range, excluding the $1380 \mathrm{~nm}$ channel which is influenced by fluctuations in water vapor. FRMS data are being acquired while viewing the RCL integrating sphere sources over a period of several months in an effort to isolate and identify any long-term source degradation characteristics. In addition to its use as a source monitor, use of the FRMS as source spatial uniformity mapper in the visible through shortwave infrared has been demonstrated. The spatial uniformity of a calibration source factors directly into absolute errors in the calibration of moderate resolution and hyperspectral imaging instruments. Lastly, use of the FRMS as a polarization insensitive detector system in the visible through shortwave infrared has also been demonstrated. The FRMS have been used not only to completely characterize the polarization of RCL source outputs but also to characterize the transmittance of flat plate polarizers.

\section{References}

[1] J.J. Butler, B.C. Johnson, S.W. Brown, H.W. Yoon, R.A. Barnes, B.L. Markham, S.F. Biggar, E.F. Zalewski, P.R. Spyak, J.W. Cooper, and F. Sakuma, “Radiometric measurement comparisons using transfer radiometers in support of the calibration of NASA's Earth Observing System (EOS) Sensors, Proc. SPIE Conference on Sensors, Systems, and Next Generation Satellites V, 3870, 180-192 (1999).

[2] J.J. Butler and R.A. Barnes, “The use of transfer radiometers in validating the visible to shortwave infrared calibrations of radiance sources used by instruments in NASA's Earth Observing System,” Metrologia, 40, S70-S77 (2003).

[3] J.J. Butler, B.C. Johnson, J.P. Rice, S.W. Brown, and R.A. Barnes, "Validation of radiometric standards for the laboratory calibration of reflected-solar Earth observing satellite instruments,” Proc. SPIE Conference on Earth Observing Systems XII, 6677, 667707-1667707-13 (2007). 
[4] B.L. Markham, J.S. Schafer, F.M. Wood Jr., P.W. Dabney, and J.L. Barker, “Monitoring large-aperture integrating sphere sources with a portable radiometer during satellite instrument calibration,” Metrologia, 35, 643-648 (1998).

[5] J.J. Butler, S.W. Brown, R.D. Saunders, B.C. Johnson, S.F. Biggar, E.F. Zalewski, B.L. Markham, P. N. Gracey, J.B. Young, and R.A. Barnes, "Radiometric measurement comparison on the integrating sphere source used to calibrate the Moderate Resolution Imaging Spectroradiometer (MODIS) and the Landsat 7 Enhanced Thematic Mapper Plus (ETM+),” J. Res. Natl. Inst. Stand. Technol., 108, 199-228 (2003).

[6] J. Marketon, P. Abel, J.J. Butler, G.R. Smith, and J.W. Cooper, “A filter radiometer monitoring system for integrating sphere sources,” Proc. SPIE Conference on Sensors, Systems, and Next Generation Satellites, 4169, 260-267 (2001).

[7] M. Johnson, "Photodetection and Measurement-Maximizing Performance in Optical Systems,” McGraw-Hill, New York, Boston \& San Francisco, 19-41, (2003). 
Figure Captions

Fig. 1 A block diagram of the FRMS.

Fig. 2 The FRMS monitoring an integrating sphere source.

Fig. 3 The FRMS detector head.

Fig. 4 The FRMS optical filter wheel.

Fig. 5 FRMS dark signals for the thermo-electrically cooled silicon + InGaAs detector.

Fig. 6 Measurements of the short-term stability of the RCL “Hardy” sphere: (a) FRMS with Si detector, 410 to 1050nm; (b) FRMS with InGaAs detector, 940 to 1640nm; (c) ASD FieldSpec3 Radiometer, 410-1640nm; (d) FRMS with extended InGaAs detector, 1050-2130nm.

Fig. 7 Measurements of the short-term stability of the RCL "Slick” sphere: (a) FRMS with Si detector, 410 to 1050nm; (b) FRMS with Ge detector, 640 to 1870nm; (c) Avantes Spectrometer, 400 to 1050 nm; (d) FRMS with extended InGaAs detector, 1050-2130nm.

Fig. 8 Measurements of the short-term stability of the RCL "Venti" sphere: (a) FRMS with Si detector, 400 to 1050nm; (b) FRMS with Ge detector, 640 to $1870 \mathrm{~nm}$.

Fig. 9 FRMS and SSBUV stability measurement comparison on the NASA Ames “ARS455” sphere: (a) $360 \mathrm{~nm}$; (b) $380 \mathrm{~nm}$.

Fig. 10 Measurements of long-term stability of the RCL "Hardy” sphere: (a) FRMS data on 4 days, 410 to 1640 nm; (b) FRMS data on 4 days normalized to 10/17/08.

Fig. 11 The RCL Source Aperture Mapper (SAM) with FRMS as detector.

Fig. 12 The RCL SAM scanning technique.

Fig. 13 FRMS SAM sphere uniformity data at 640nm: (a) "Hardy” sphere; (b) “Slick” sphere; (c) "Venti” sphere; (d) “Grande” sphere. The graduations on the $\mathrm{x}$ and y axes indicate centimeters. Fig. 14 Polarization measurement setup showing, from left to right, an integrating sphere, the flat sheet polarizer in a rotation stage, the glass optical flat tilted $30^{\circ}$ to the primary optical axis of the setup, and the FRMS.

Fig. 15 FRMS signal in volts as a function of the polarization angle of the flat sheet polarizer. For these data, the FRMS viewed an integrating sphere through the sheet polarizer and a glass optical flat tilted $30^{\circ}$ to the primary optical axis.

Fig. 16 Instability of the dark signal of the FRMS equipped with the thermo-electrically cooled InGaAs detector. 
Fig. 17 Chopper installed before the filter wheel in the FRMS to enable AC measurements using a lock-in amplifier.

Fig. 18 Comparison of FRMS measurements made in DC and AC operating modes using the RCL "Grande" sphere as a source: (a) FRMS with extended InGaAs detector viewing the sphere with 8300W and 1-150W lamp illuminated (i.e. full lamp level); (b) FRMS with extended InGaAs detector viewing the sphere with 4-300W lamps illuminated (i.e. lower lamp level). 
Table 1. Detectors used in the FRMS

\begin{tabular}{|c|c|c|c|c|c|c|c|c|}
\hline \multicolumn{2}{|c|}{ Detector Element } & Cooling & $\begin{array}{c}\text { Active } \\
\text { Area } \\
\text { Size } \\
(\mathrm{mm}) \\
\end{array}$ & $\begin{array}{c}\text { Spectral } \\
\text { Response } \\
\text { Range } \\
(\mathrm{nm})\end{array}$ & $\begin{array}{c}\text { Sensitivity } \\
@ \lambda_{\mathrm{P}} \\
(\mathrm{A} / \mathrm{W})\end{array}$ & $\begin{array}{c}\text { Dark } \\
\text { Current }\end{array}$ & $\begin{array}{c}\text { Shunt } \\
\text { Resistance } \\
\mathrm{R}_{\mathrm{sh}}\end{array}$ & $\begin{array}{c}\mathrm{NEP} \\
@ \lambda_{\mathrm{P}} \\
\left(\mathrm{W} / \mathrm{Hz}{ }^{\mathrm{P}}{ }^{1 / 2}\right)\end{array}$ \\
\hline \multicolumn{2}{|c|}{$\mathrm{Si}$} & $\begin{array}{l}\text { One-stage } \\
\text { TE-cooled } \\
\left(-10^{\circ} \mathrm{C}\right)\end{array}$ & $2.4 \times 2.4$ & $190-1100$ & 0.5 & $10 \mathrm{pA}^{\mathrm{a}}$ & $1 \mathrm{G} \Omega^{\mathrm{a}}$ & $8.1 \times 10^{-15 b}$ \\
\hline \multicolumn{2}{|c|}{ Extended-InGaAs } & $\begin{array}{c}\text { Two-stage } \\
\text { TE-cooled } \\
\left(-20^{\circ} \mathrm{C}\right)\end{array}$ & ØЗ & $\begin{array}{c}1200- \\
2570\end{array}$ & 1.3 & $7.5 \mu \mathrm{A}^{\mathrm{c}}$ & $6 \mathrm{~K} \Omega^{\mathrm{a}}$ & $1.8 \times 10^{-12 b}$ \\
\hline \multirow[t]{2}{*}{$\begin{array}{c}\text { Two- } \\
\text { element } \\
\text { Detector }\end{array}$} & $\mathrm{Si}$ & $\begin{array}{c}\text { One-stage } \\
\text { TE-cooled } \\
\left(-10^{\circ} \mathrm{C}\right)\end{array}$ & $2.4 \times 2.4$ & $320-1670$ & 0.45 & $50 \mathrm{pA}^{\mathrm{a}}$ & $200 \mathrm{M} \Omega^{\mathrm{a}}$ & \\
\hline & InGaAs & $\begin{array}{c}\text { One-stage } \\
\text { TE-cooled } \\
\left(-10^{\circ} \mathrm{C}\right)\end{array}$ & $\varnothing 1$ & & 0.55 & $70 \mathrm{pA}^{* 3}$ & $1.5 \mathrm{G} \Omega^{\mathrm{a}}$ & \\
\hline \multirow[t]{2}{*}{$\begin{array}{c}\text { Extended } \\
\text { Two- } \\
\text { element } \\
\text { Detector }\end{array}$} & $\mathrm{Si}$ & $\begin{array}{c}\text { One-stage } \\
\text { TE-cooled } \\
\left(-10^{\circ} \mathrm{C}\right)\end{array}$ & $2.4 \times 2.4$ & $320-2570$ & 0.45 & $50 \mathrm{pA}^{\mathrm{a}}$ & $200 \mathrm{M} \Omega^{\mathrm{a}}$ & \\
\hline & InGaAs & $\begin{array}{c}\text { One-stage } \\
\text { TE-cooled } \\
\left(-10^{\circ} \mathrm{C}\right)\end{array}$ & $\varnothing 1$ & & 0.60 & $1.5 \mu \mathrm{A}^{\mathrm{c}}$ & $30 \mathrm{~K} \Omega^{\mathrm{a}}$ & \\
\hline \multicolumn{2}{|c|}{$\mathrm{Ge}$} & $\begin{array}{c}\text { Two-stage } \\
\text { TE-cooled } \\
\left(-20^{\circ} \mathrm{C}\right)\end{array}$ & $\varnothing 5$ & $800-1800$ & 0.8 & $30 \mathrm{nA}^{\mathrm{a}}$ & $330 \mathrm{~K} \Omega^{\mathrm{a}}$ & $3.0 \times 10^{-13 b}$ \\
\hline
\end{tabular}

${ }^{\mathrm{a}} 25^{\circ} \mathrm{C}, \mathrm{V}_{\mathrm{R}}=10 \mathrm{mV}$

${ }^{\mathrm{b}} 25^{\circ} \mathrm{C}, \mathrm{V}_{\mathrm{R}}=0 \mathrm{~V}$

${ }^{\mathrm{c}} 25^{\circ} \mathrm{C}, \mathrm{V}_{\mathrm{R}}=1 \mathrm{~V}$

Table 2. FRMS noise equivalent radiance, in $\times 10^{-2} \mathrm{~W} / \mathrm{m}^{2} \mathrm{sr} \mu \mathrm{m}$, for select detectors and bands between 410 and $2130 \mathrm{~nm}$ with the FRMS operated in DC mode.

\begin{tabular}{|c|c|c|c|c|c|c|c|c|c|c|c|}
\hline Wavelength (nm) & 410 & 440 & 460 & 640 & 840 & 1050 & 1240 & 1380 & 1640 & 1870 & 2130 \\
\hline Si detector & 0.45 & 0.82 & 0.94 & 0.99 & 0.83 & 0.57 & & & & & \\
\hline Ge detector & & & & & 5.79 & 3.46 & 2.33 & 2.22 & 1.82 & & \\
\hline InGaAs detector & & & & & & 11.5 & 4.74 & 3.81 & 1.27 & 4.67 & 2.95 \\
\hline
\end{tabular}

Table 3. Percent difference between polarization measurements using a $30^{\circ}$ tilted glass plate, a flat plate polarizer, and the FRMS and the theoretical Fresnel prediction

\begin{tabular}{|c|c|c|c|c|c|c|c|c|c|c|c|}
\hline Wavelength (nm) & 360 & 380 & 410 & 440 & 460 & 550 & 640 & 780 & 840 & 870 & 1050 \\
\hline Difference (\%) & 0.234 & 0.207 & 0.171 & 0.150 & 0.128 & 0.124 & 0.014 & 0.137 & 0.085 & 0.092 & 0.362 \\
\hline
\end{tabular}




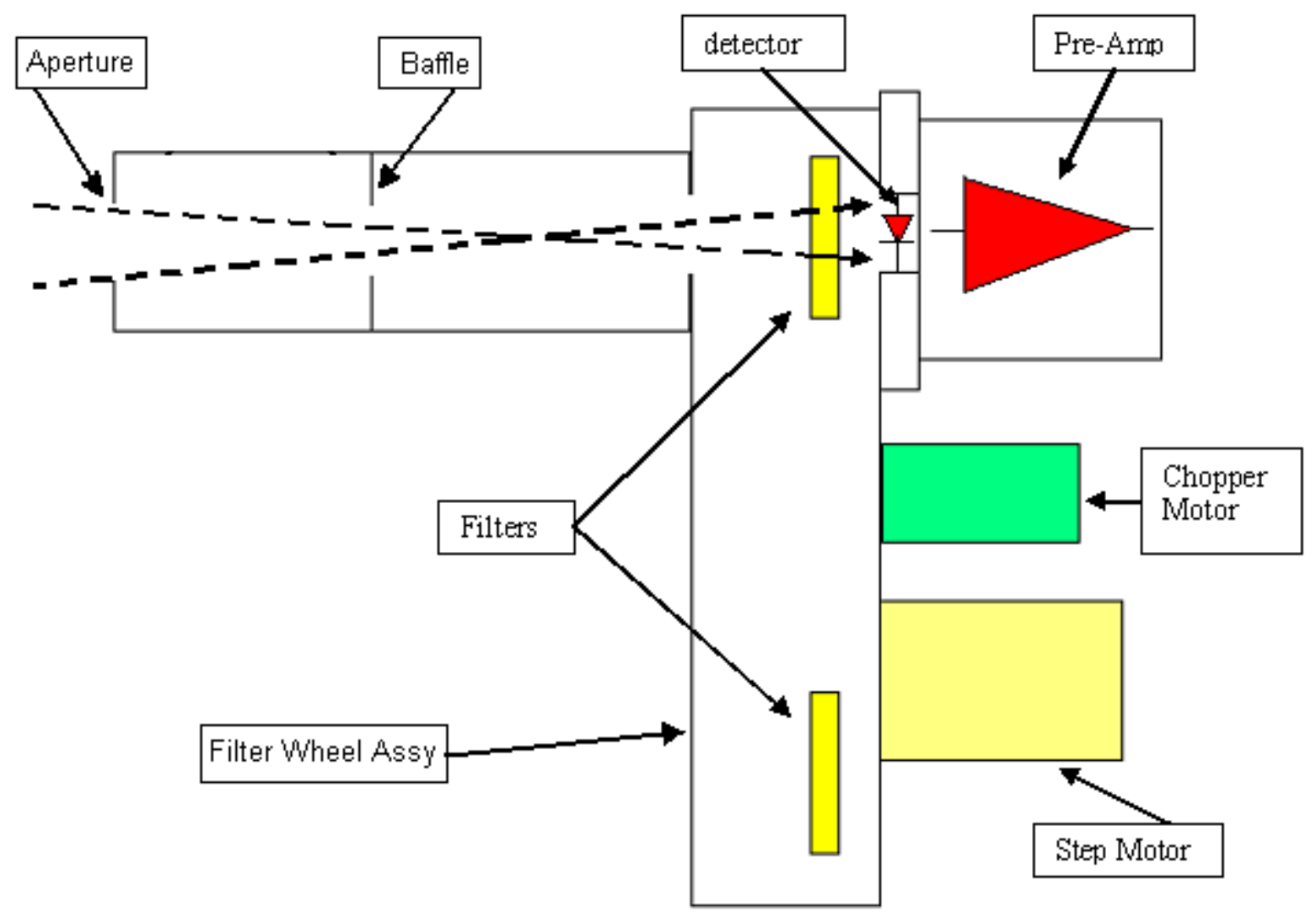

Figure 1 


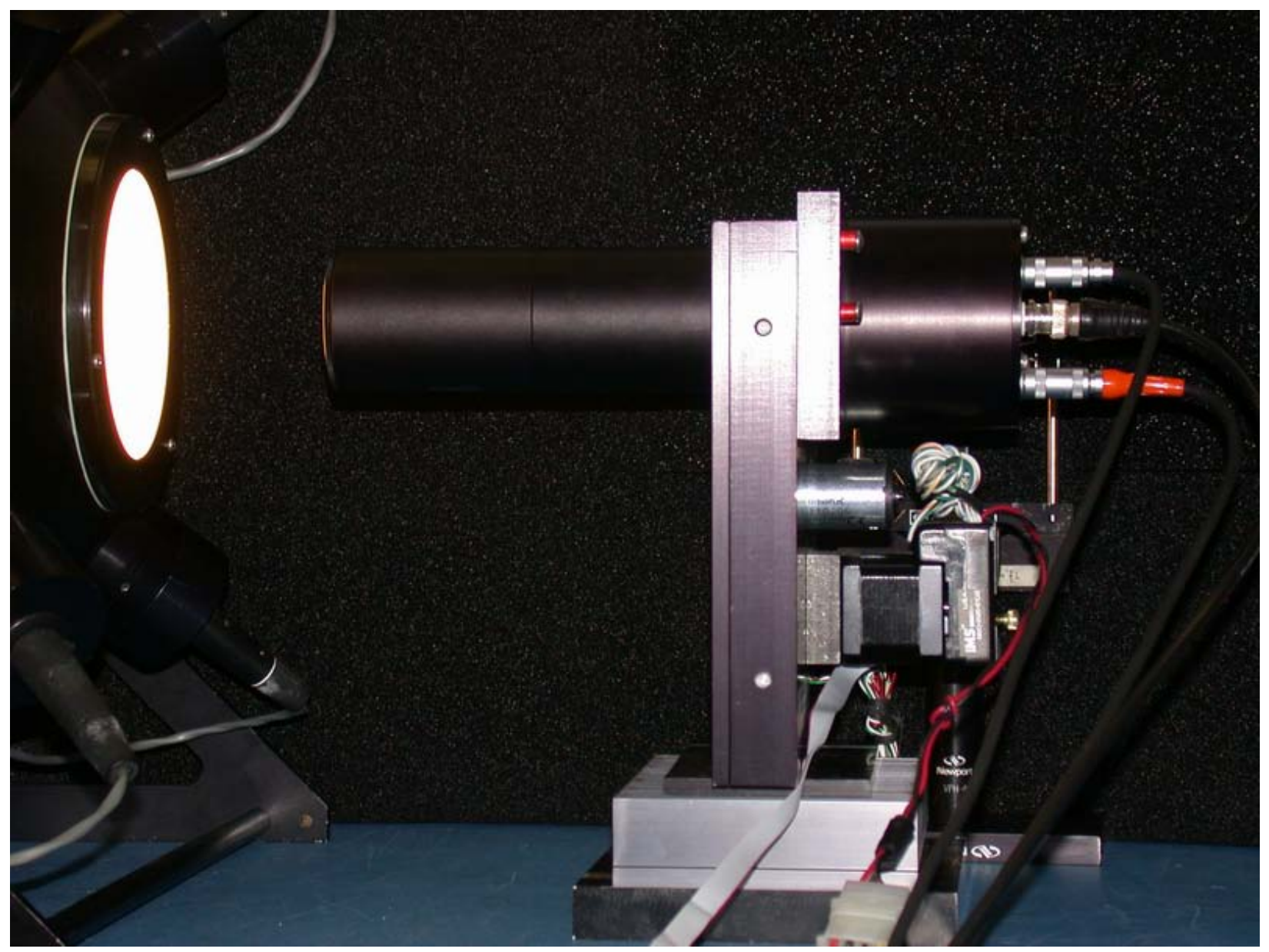

Figure 2 


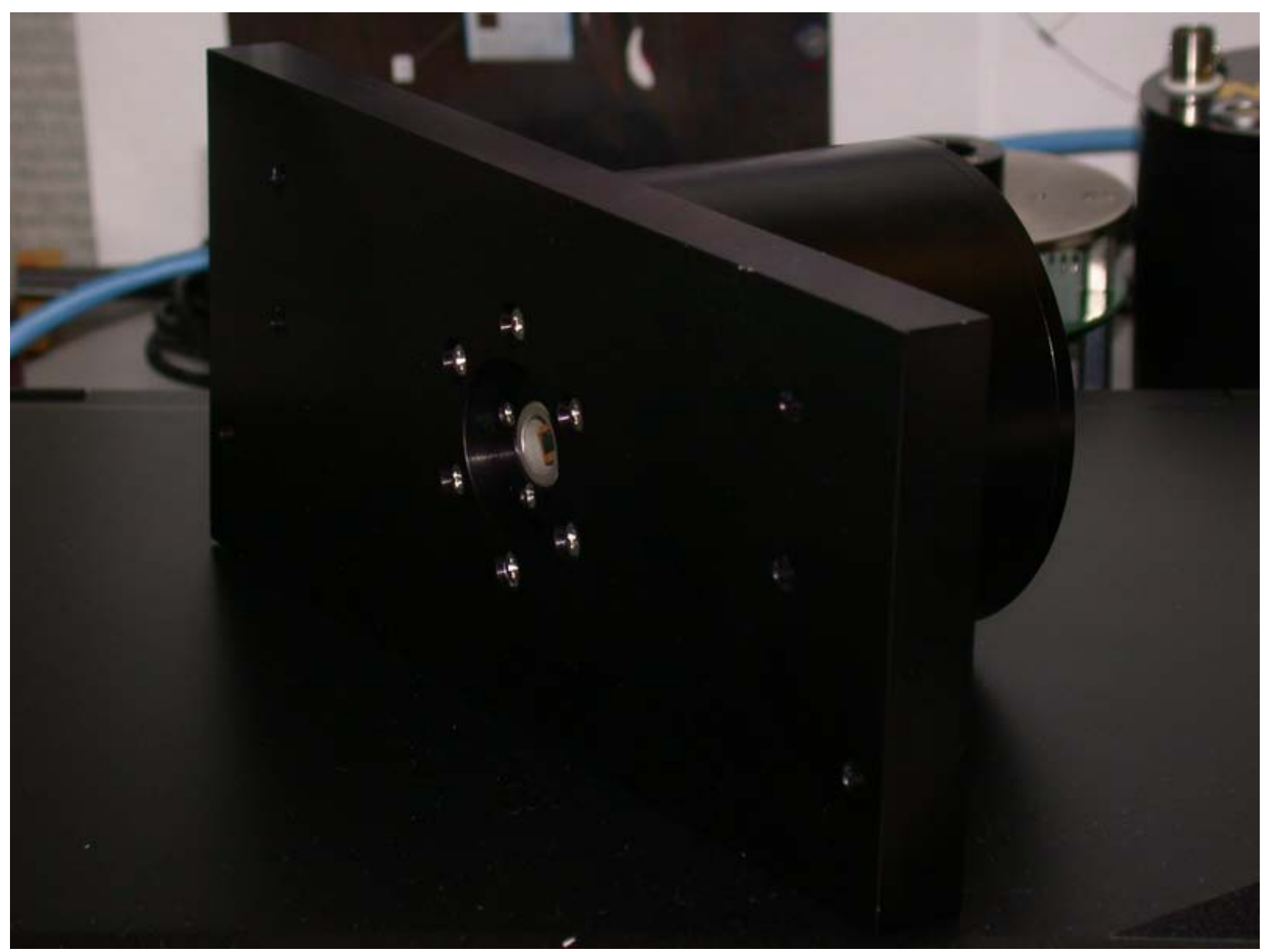

Figure 3 


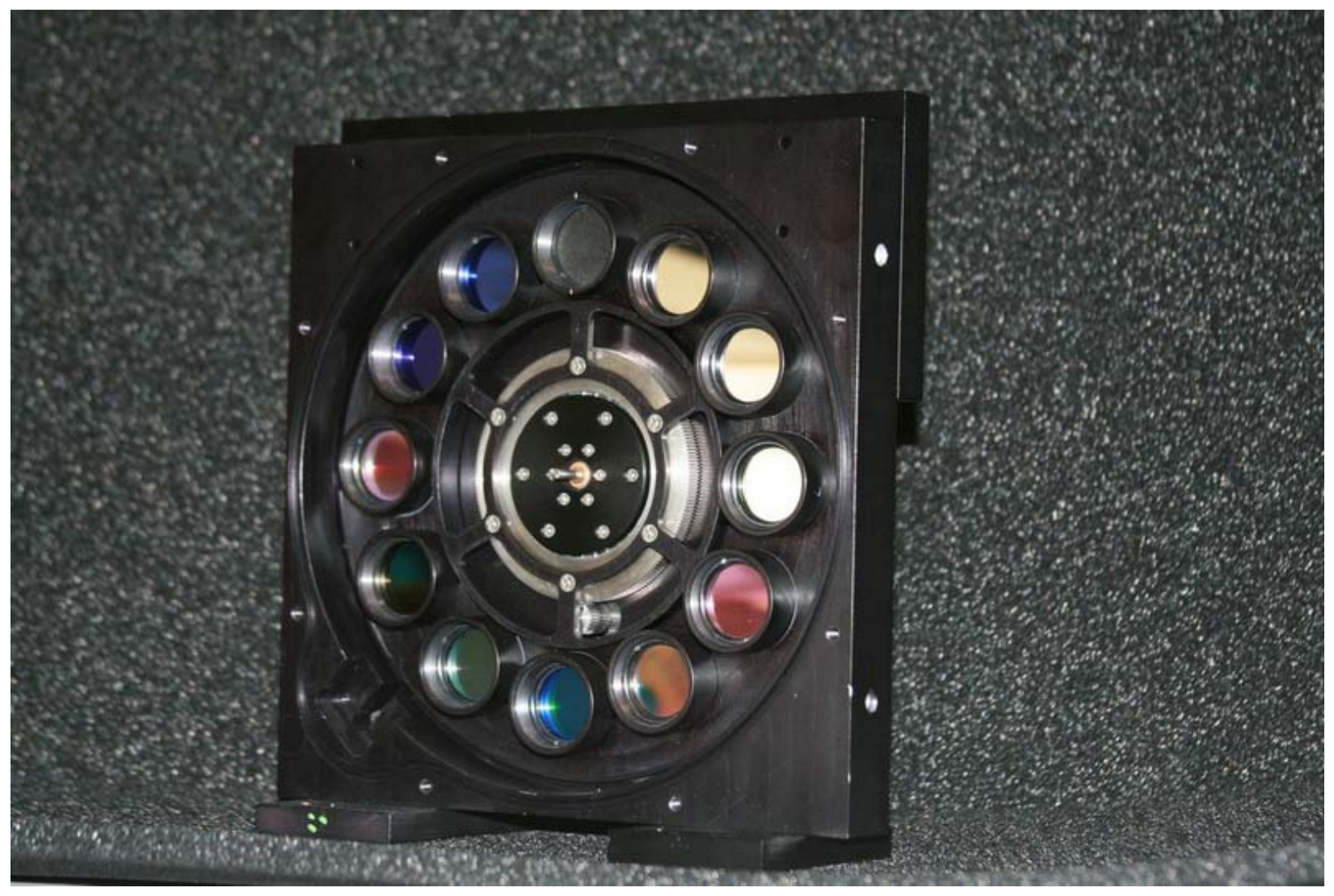

Figure 4 


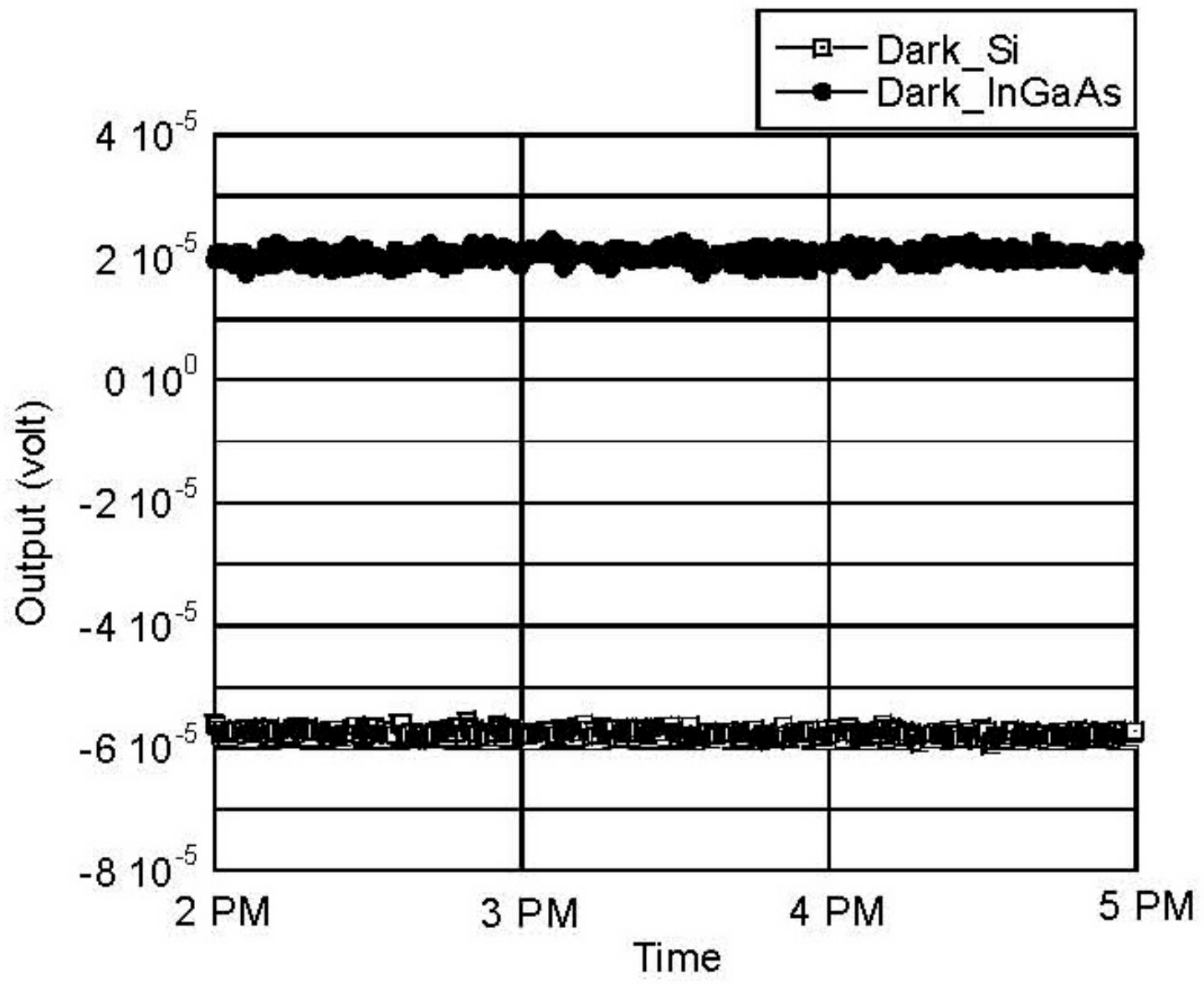

Figure 5 

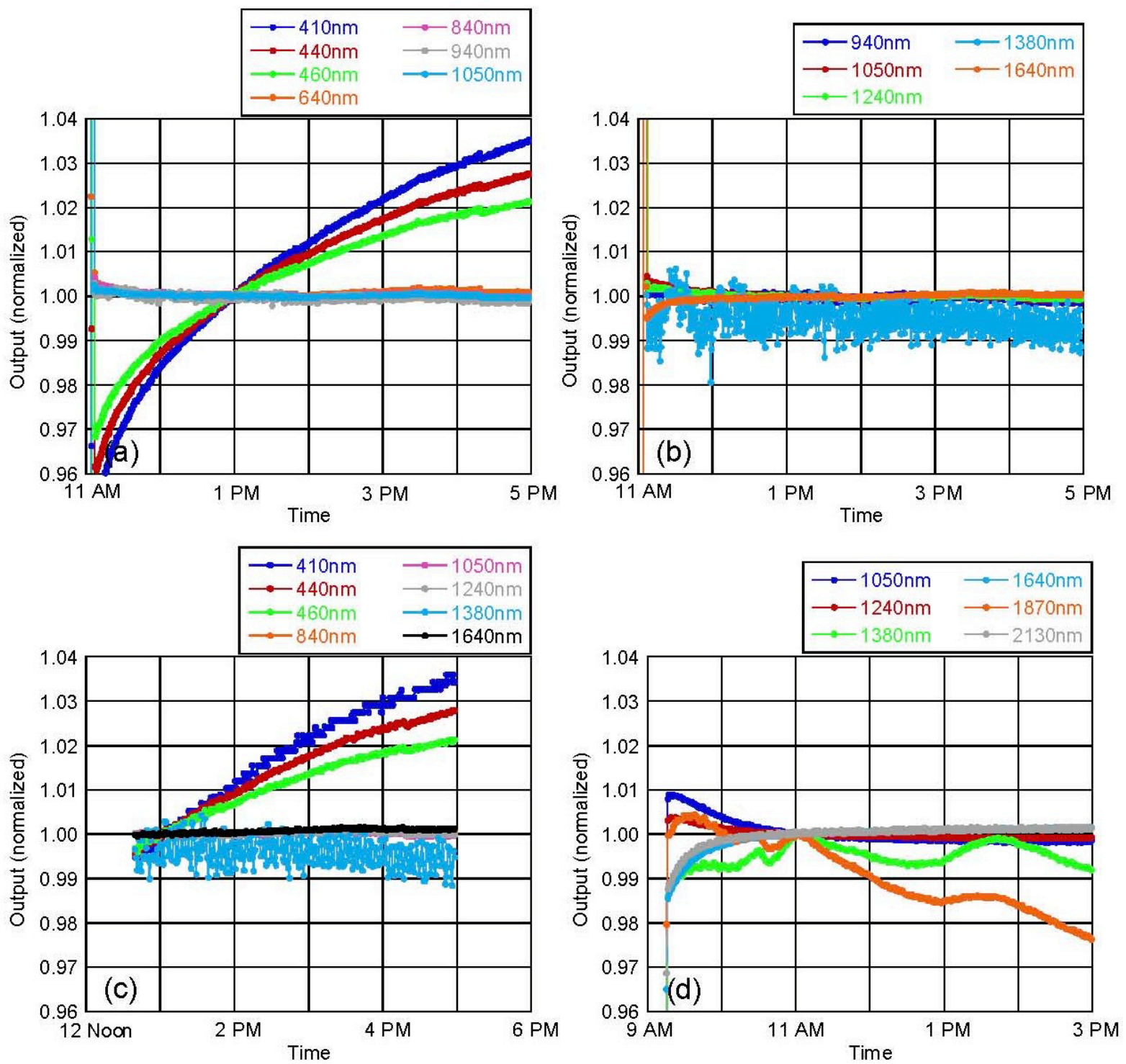

Figure 6 

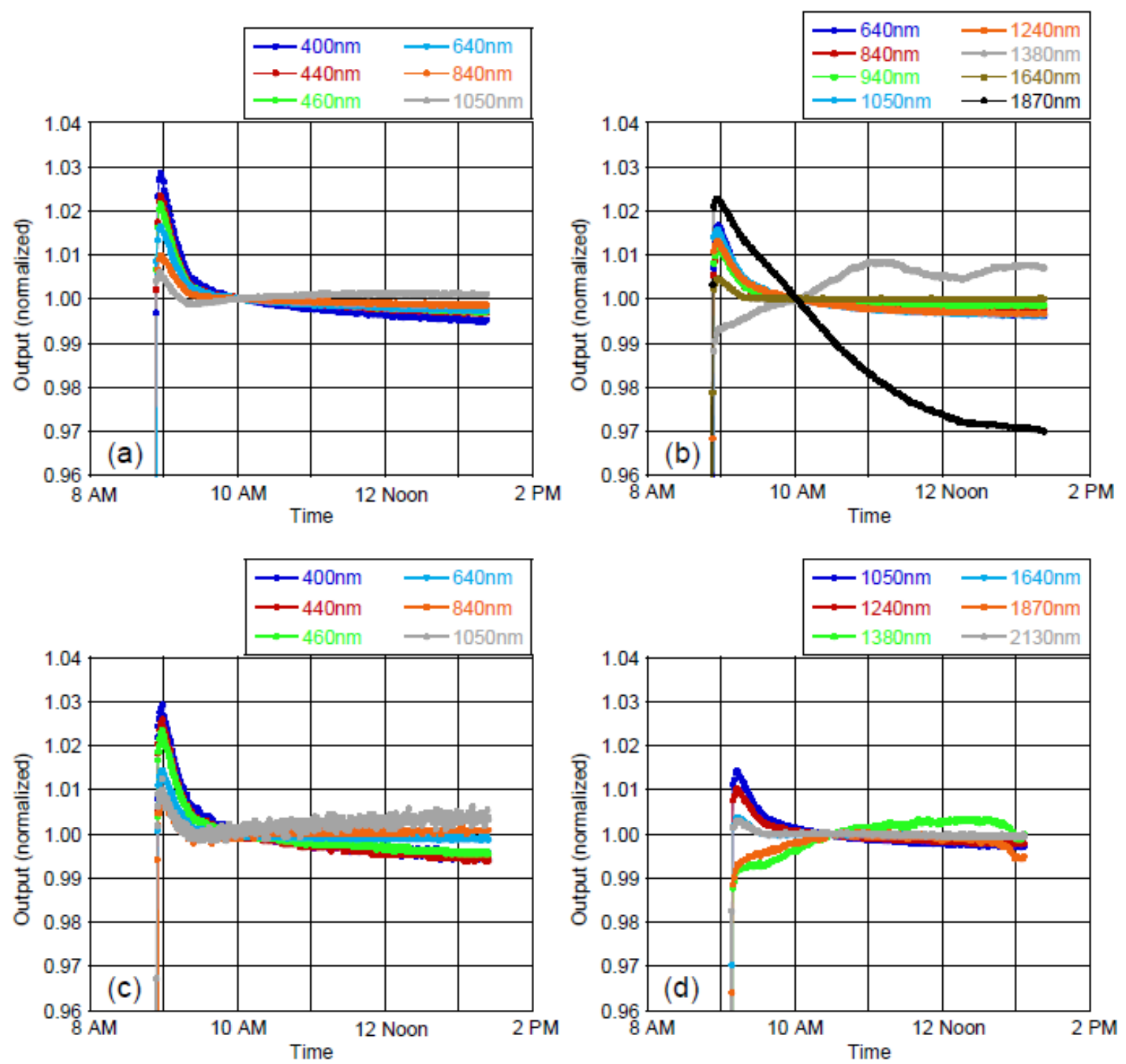

Figure 7 

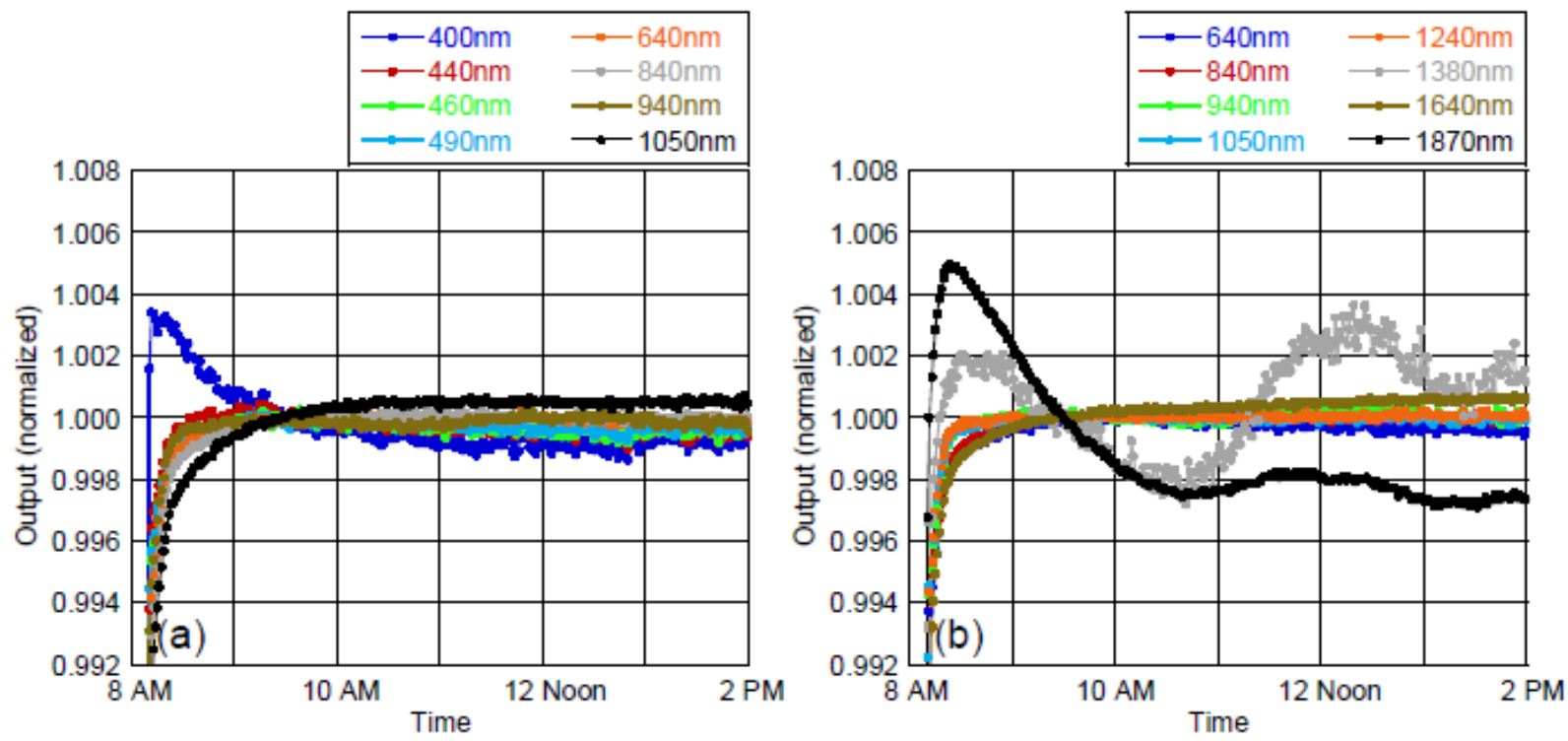

Figure 8 

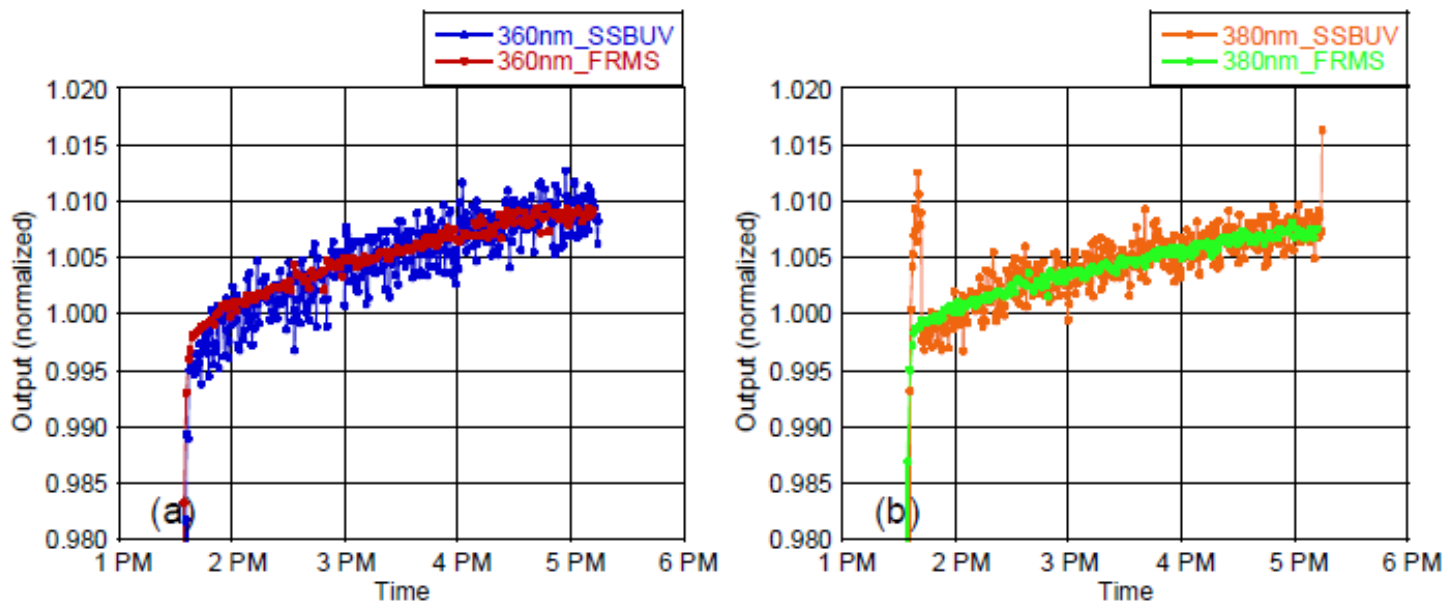

Figure 9 

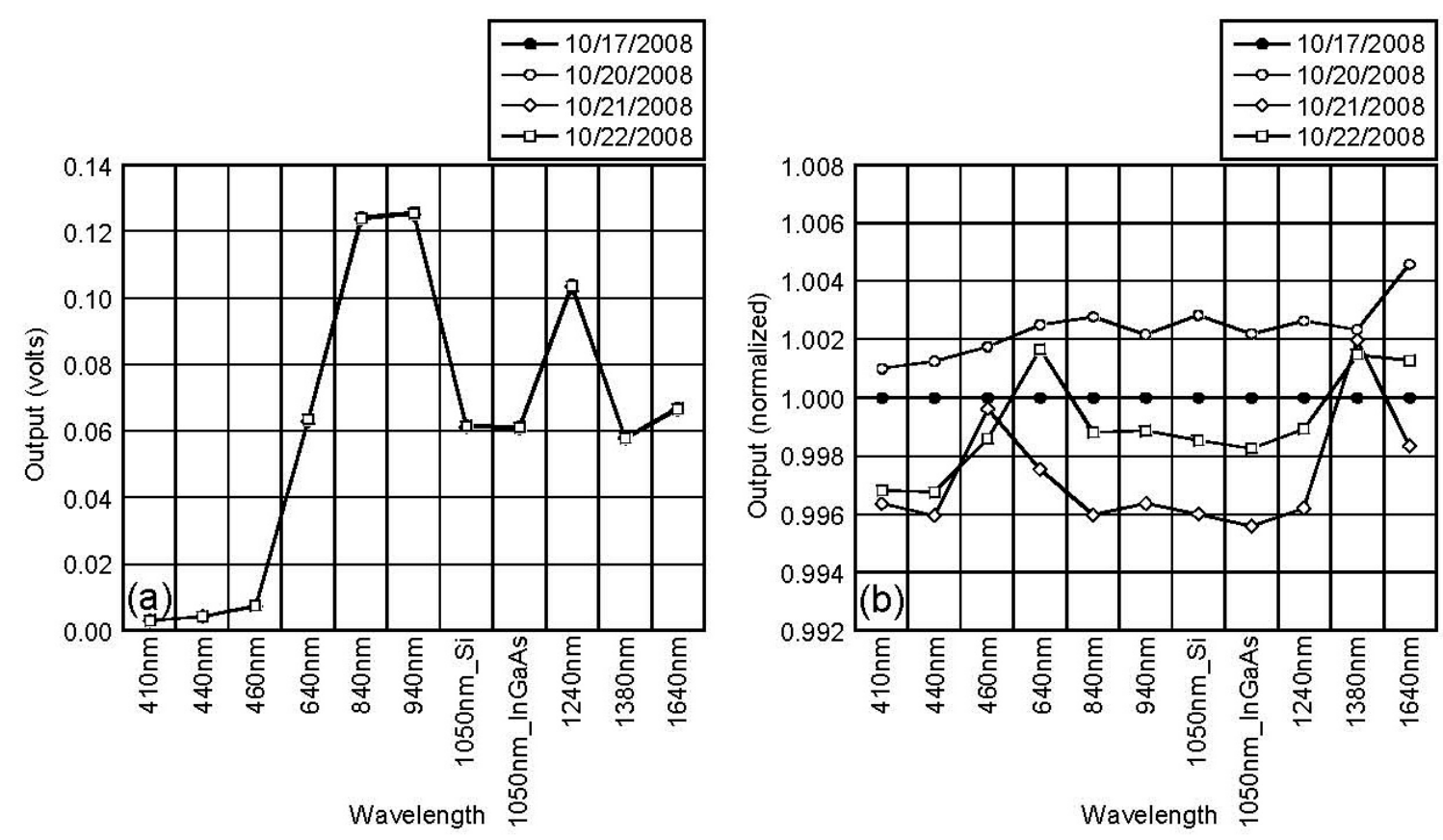

Figure 10 


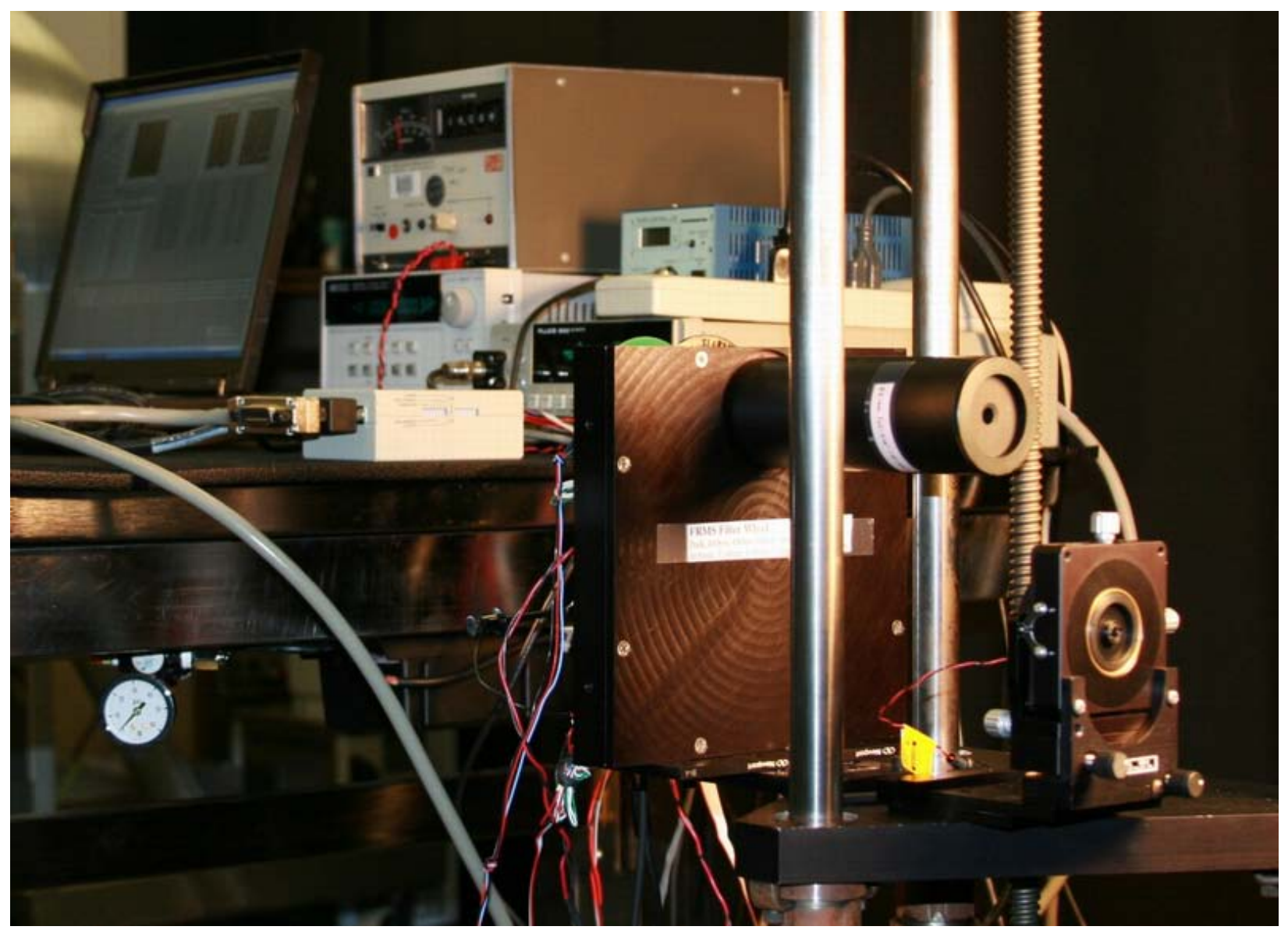

Figure 11 


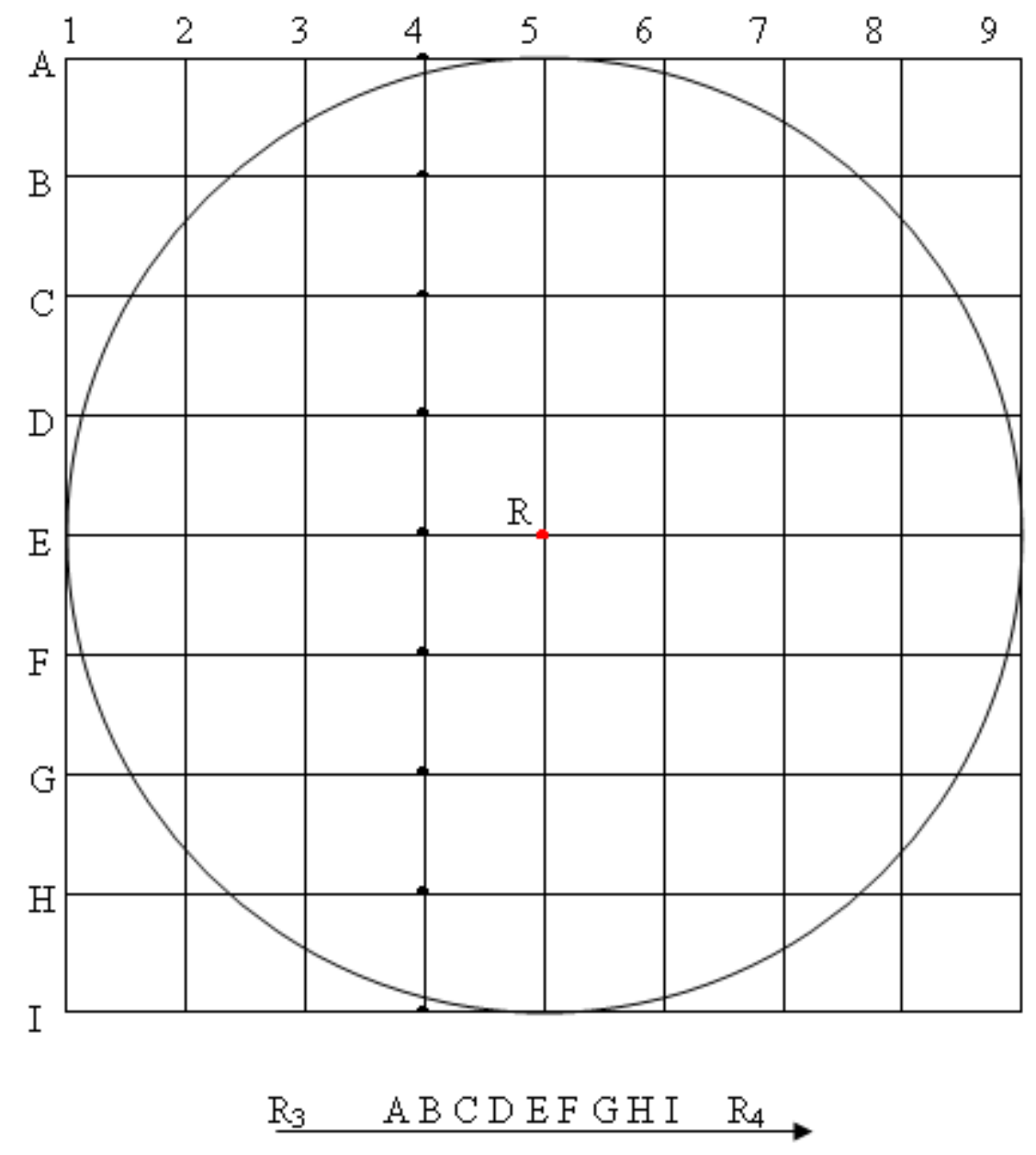

Figure 12 


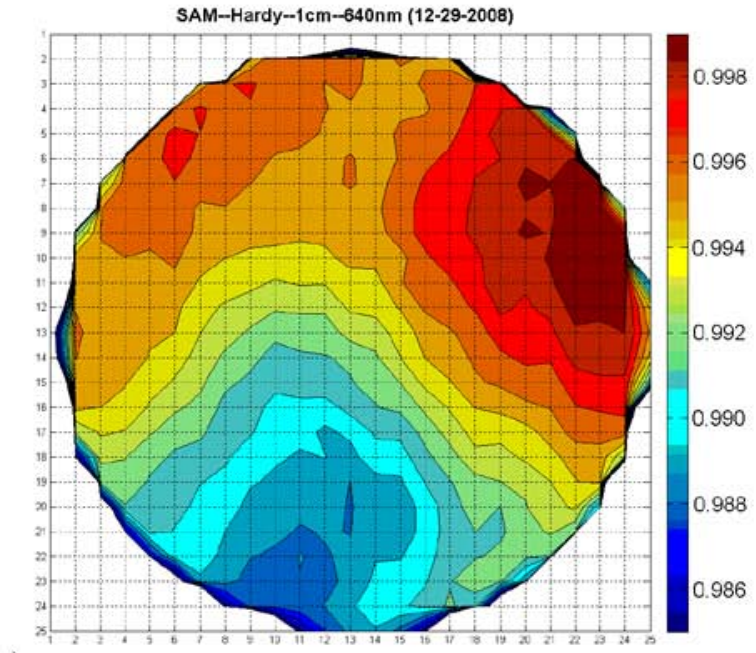

(a)

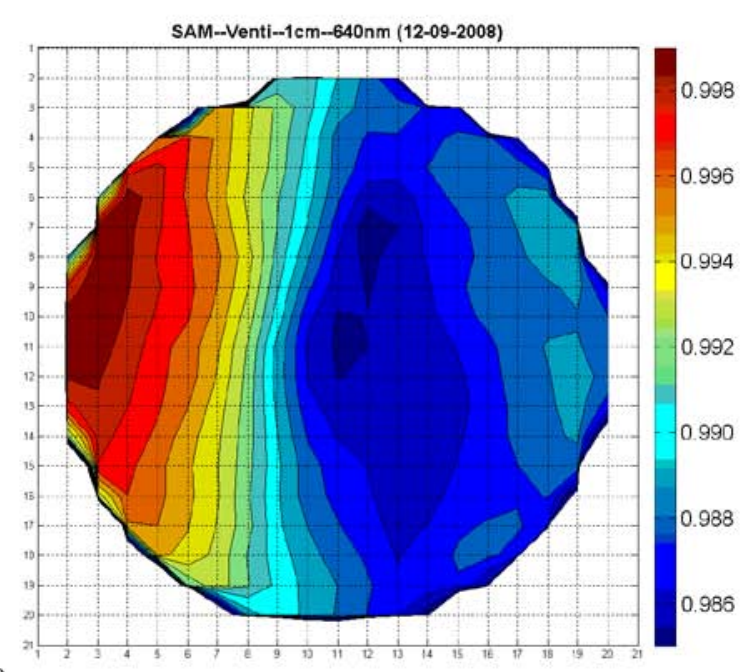

(c)

Figure 13

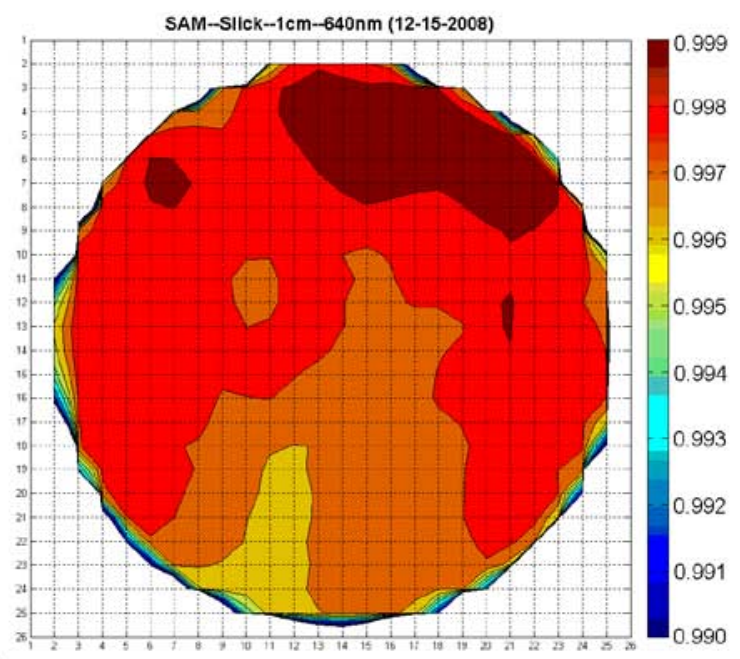

(b)

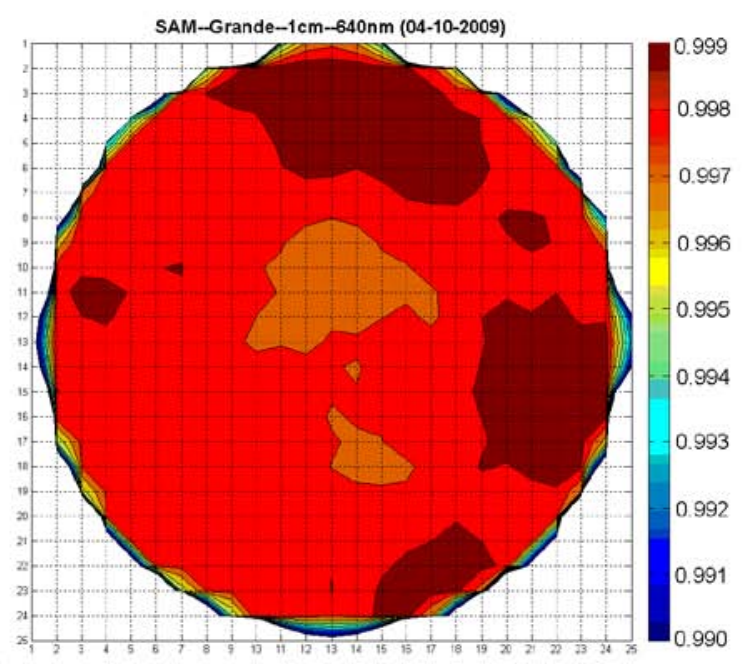

(d) 


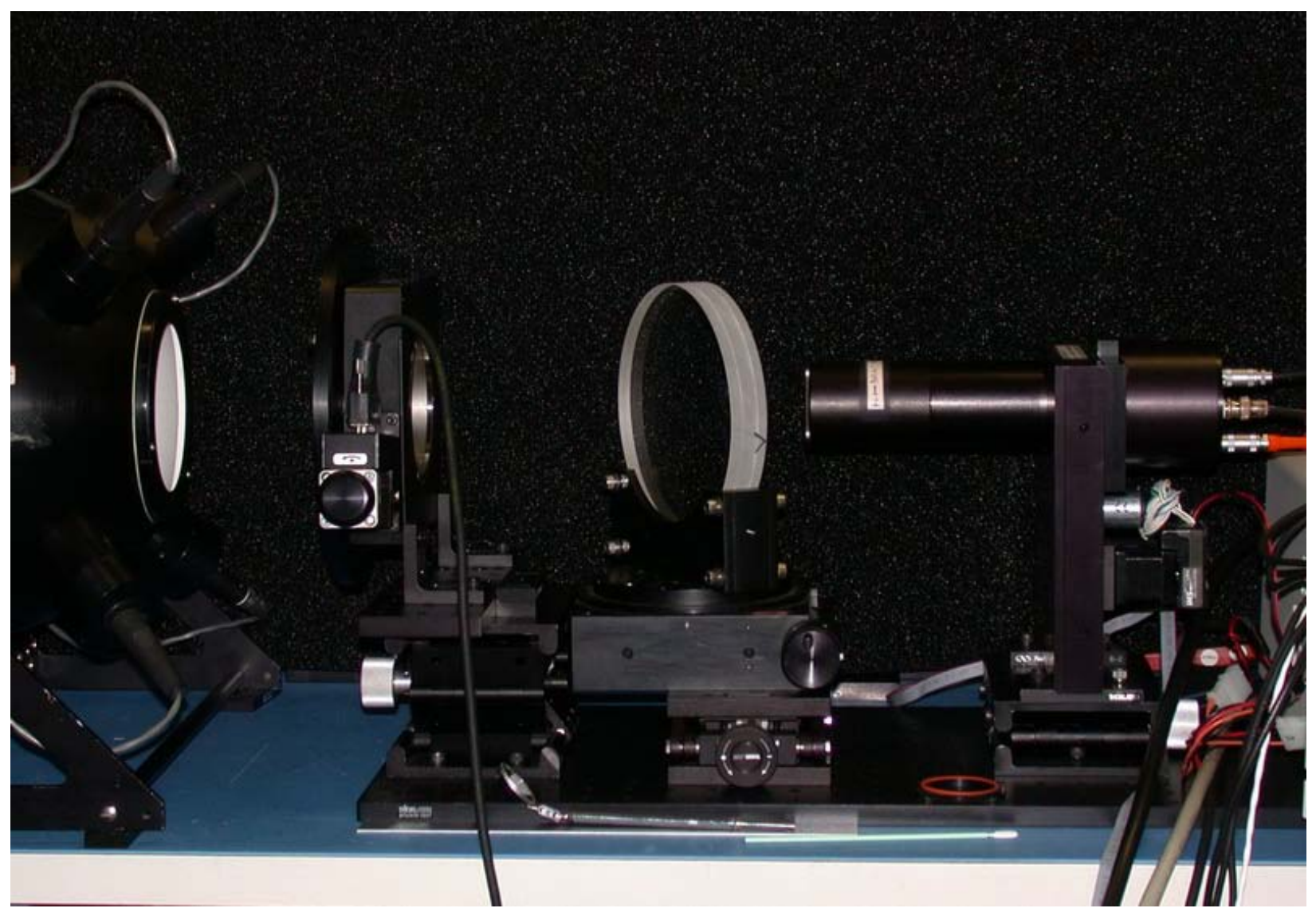

Figure 14 


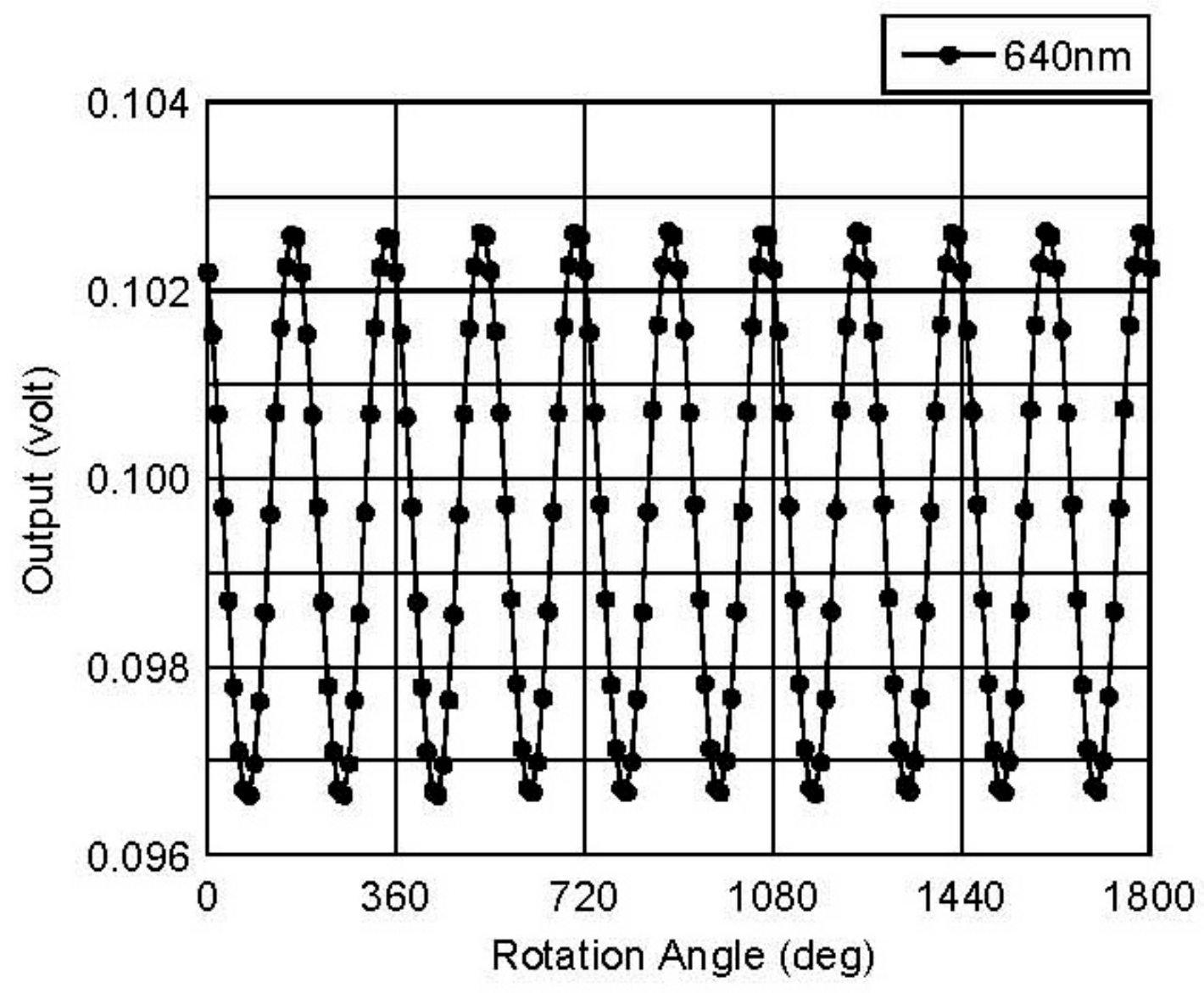

Figure 15 


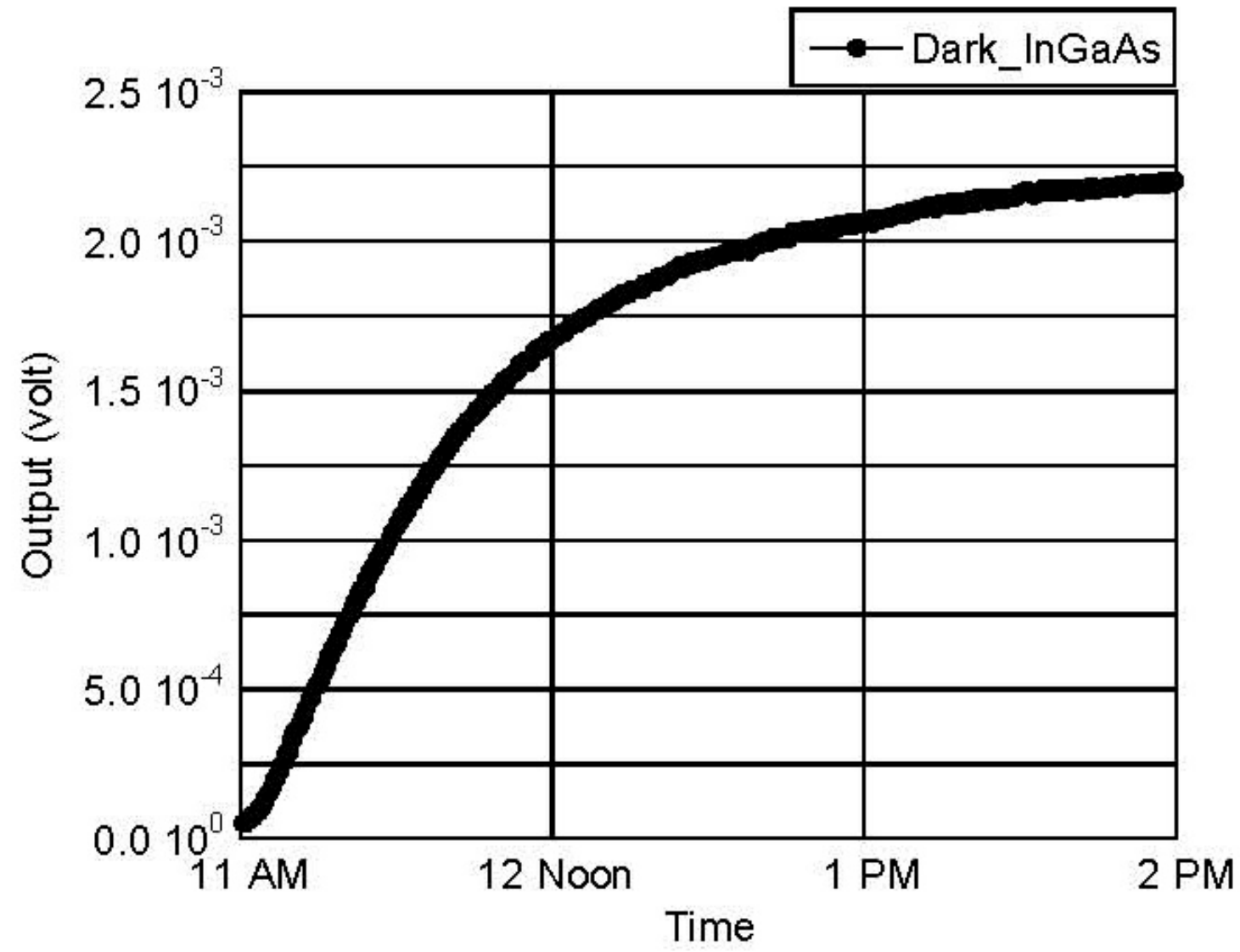

Figure 16 


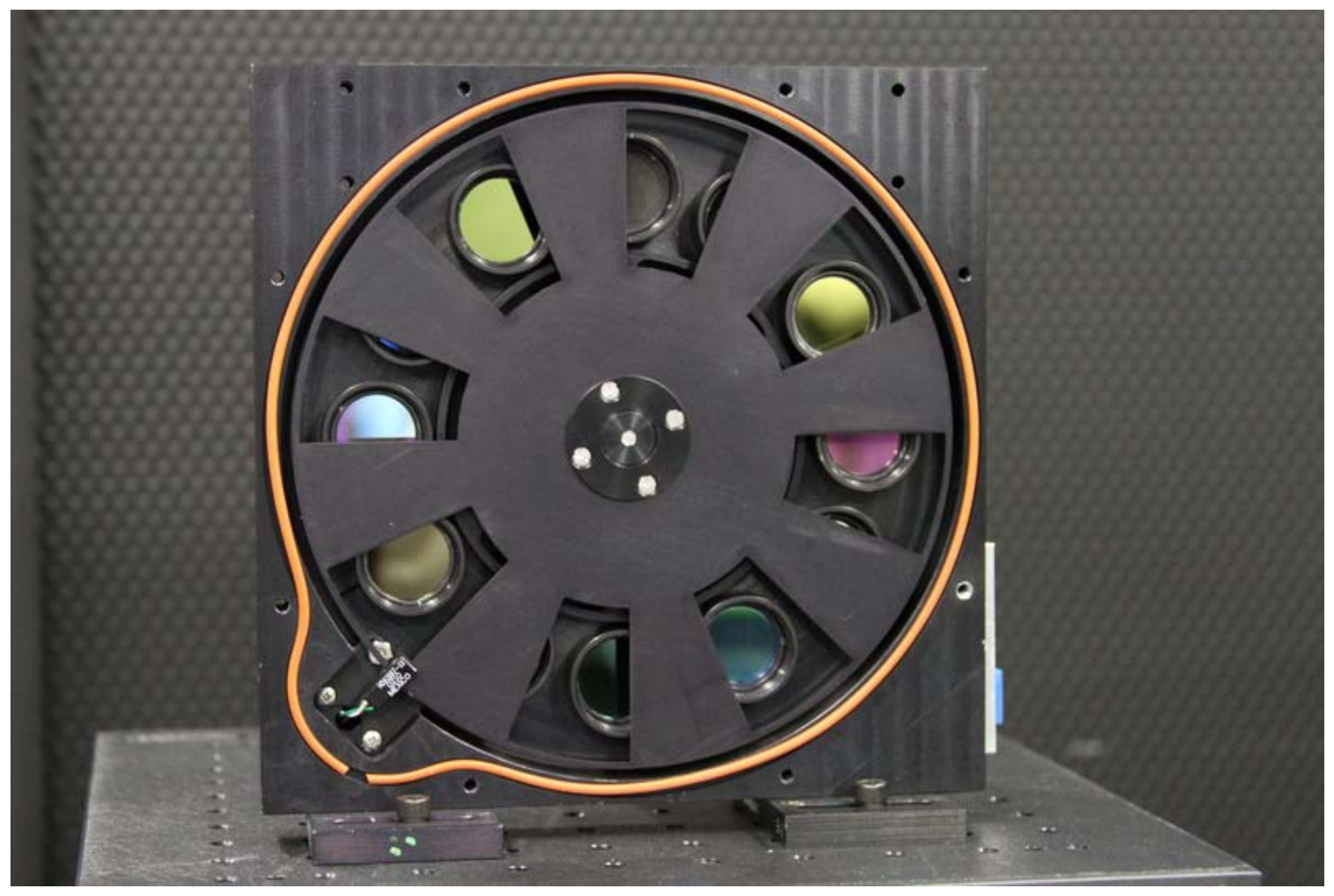

Figure 17 

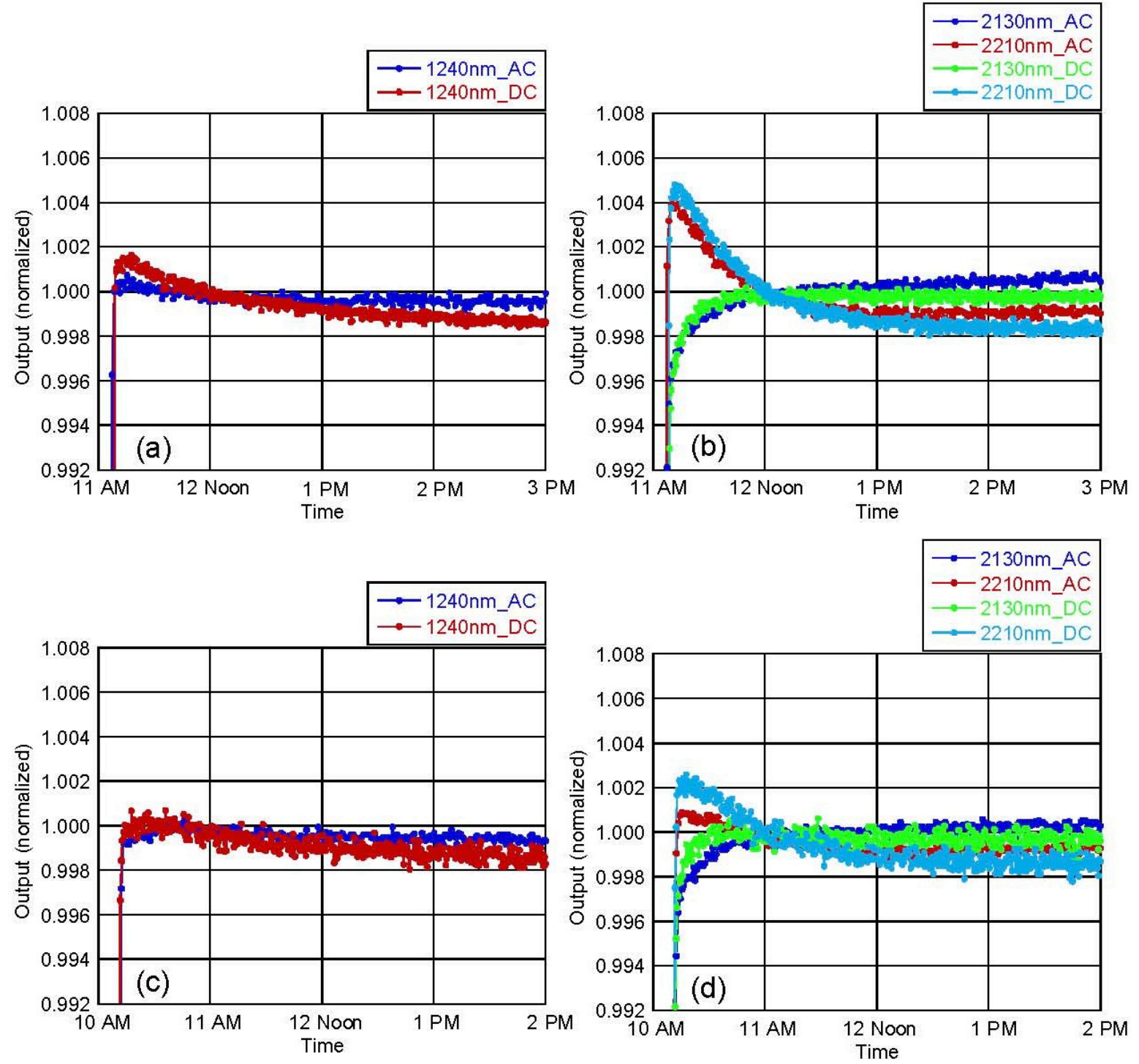

Figure 18 